\title{
LESSON 128
}

Statement 6

TARGET TIME: 10 minutes.

Use your statement of account framework as a backing sheet as you type this.

Invoice 8

TARGET TIME: 10 minutes.

Use your invoice framework as a backing sheet as you type this.

Memo 7

TARGET TIME: 5 minutes.

Type on a suitable form.

\section{Tabulation 35}

PAPER: A5 $(210 \times 148 \mathrm{~mm})$.

TARGET TIME: 10 minutes.

Rule up.

UNIT 32

From Nouman \& Holmes Ltd, 10 Bumlay Road, Manchester. MAS ICC To Neald \& Millerchip Lid, 56 Sandurell Road, Blackpool. LA9 3 BB 8th July, 19.. Ref. NM/230

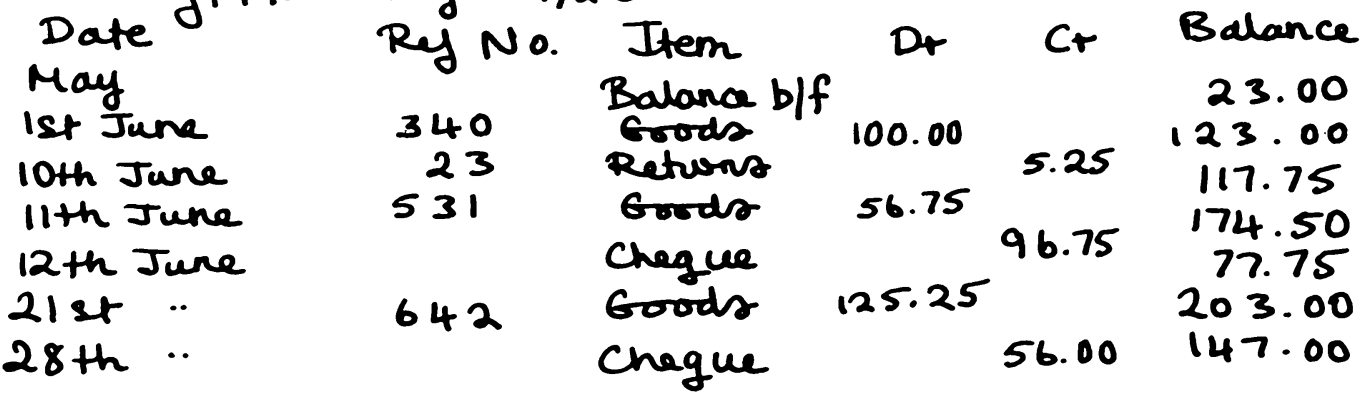

From: J. Kendrick Ltd., 34 Dyke Lane, Stourport. WO7 2ST

To: C. J. Berry Ltd., 86 Brook Street, Stourbridge. ST3 6ES To-day's date Invoice No. 861 Carrier B.R.S. Packets 7

\begin{tabular}{lllr} 
Quantity & \multicolumn{1}{c}{ Description } & Price & \multicolumn{1}{c}{ Total } \\
2 tons & Plate maize & 25.75 & 51.50 \\
4 tons & Feeding barley & 26.25 & 105.00 \\
5 tons & Wheat & 28.00 & 140.00 \\
1 ton & Concentrate & 51.75 & 51.75 \\
1 ton & Sharpes & 15.00 & 15.00 \\
\hline
\end{tabular}

$£ 363.25$

From Manager

To All sales stoff

There will ve a meeting for all staff next Monday at 1400 tours in my office to discuss the forthcoming sale. Whel Heads of Dapartment please bring ulong wh their plans together. with extra staff requirments and advertiong suggestions.

\begin{tabular}{|l|r|r|r|}
\hline \multirow{2}{*}{$\begin{array}{c}\text { Mode } \\
\text { of }\end{array}$} & \multicolumn{3}{|c|}{ Subject Entry } \\
\cline { 2 - 5 } Examination & Boys & Girlo & Total \\
\hline Mode 1 & 34,400 & 38,226 & 72,626 \\
Mode 2 & 506 & 309 & 815 \\
Mode 3 & 326 & 125 & 451 \\
\hline Total & 35,232 & 38,660 & 73,892 \\
\hline
\end{tabular}

